\title{
Multimodal Neural Block Analgesia Versus Morphine Analgesia After Elective Knee Surgery
}

\author{
Dragana Lončar-Stojiljković ${ }^{1}$
}

\section{ABSTRACT}

Background: Total knee arthroplasty has become a standard for treatment of endstage knee osteoarthritis. Due to intense and complex knee innervation, there is a need to improve the anaesthetic/analgesic approach to such operations. The aim of this randomised clinical trial was to compare the analgesic efficacy of the classical regimen and two of those based on the nerve blocks.

Methods: A total of 60 patients was included and subjected to elective total knee arthroplasty under the general balanced anaesthesia. They were randomised to receive postoperatively (1) only morphine 5-10 mg q6h and paracetamol $1 \mathrm{~g} \mathrm{q} 6 \mathrm{~h}$ (MP), (2) femoral nerve block (FNB) or (3) fascia iliac compartment nerve block (FICNB). Nerve blocks were produced by a single administration of $30-40 \mathrm{ml}$ of bupivacaine $0.5 \%$. Pain intensity, duration of neural block and additional consumption of analgesics were recorded postoperatively.

Results: There were no demographic differences among the three groups of patients. Pain intensity was significantly lower in the two nerve block groups than in the MP group. The same two groups also demanded significantly less analgesics postoperatively than the MP group. Cardiovascular control was significantly better in the nerve block groups. There were no significant differences between the FNB and FICNB groups of patients regarding any of the studied parameters.

Conclusion: In comparison with the classical MP analgesia, use of FNB or FICNB after the elective total knee arthroplasty results in lower pain scores, lower systolic blood pressure and less consumption of analgesics in the immediate postoperative course.

Key words: knee arthroplasty, anaesthesia, nerve block, morphine, paracetamol.

\section{INTRODUCTION}

Osteoarthritis (OA) is the leading form of arthritis in humans and is responsible for suffering of the $15-18 \%$ of the population. ${ }^{1}$ According to the Danish registry of chronic diseases, the prevalence of the knee localisation of OA is $3.9 \%{ }^{2}$ According to the Korean study performed in patients aged $>50$ who underwent knee radiography, prevalence of knee OA was $13 \%$ and it was positively associated with risk factors, such as older age, female gender, obesity, hypertension, low educational level, and infrequent strength exercises. $^{3}$

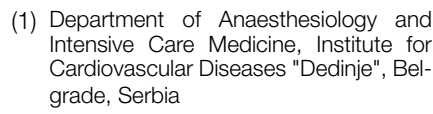

(1) Department of Anaesthesiology and Intensive Care Medicine, Institute for Cardiovascular Diseases "Dedinje", Belgrade, Serbia

Correspondence:

DRAGANA

LONČAR-STOJILJKOVIĆ

E: draganalost@gmail.com

\section{ARTICLE INFO}

Received: 27 August 2019

Revision received: 17 September 2019 Accepted: 19 September 2019

Copyright $\odot 2019$ Lončar-Stojiliković. This is an open access article distributed under the Creative Commons Attribution License (CC BY), which permits unrestricted use, distribution, and reproduction in any medium, provided the original work is properly cited. 
activities. ${ }^{11}$ Several non-pharmacological (body weight loss, muscle strength-increasing exercises, physiotherapy) and pharmacological regimens (non-steroidal anti-inflammatory drugs po or im, intraarticular injections of viscosupplementation with hyaluronic acid, corticosteroids or biologicals) are used to treat this very painful and disabling condition. ${ }^{12}$

Total knee arthroplasty (TKA) remains an ultimum refugium for the patients with end-stage osteoarthritis and its prevalence is also on the rise, along with the prevalence of OA. In the UK study, the indication for TKA was made in $2.04 \%$ of people older than 55 with knee problems. ${ }^{13}$ In Canada, for example, there was a 5-year increase in the use of TKA over the last five years of $16 \% .^{14}$ It is projected that between the 2005 and 2030 the number of performed TKAs in the US will increase $673 \%$ to 3.5 million procedures annually and if the revision operations are taken into consideration, the overall increase is expected to be over eight-fold ${ }^{15}$. Additional explanation for this phenomenon is in the fact that TKAs, despite the increased rate of postoperative complications, represent a rational solution for the end-stage knee OA even in the population aged over $80 .{ }^{16}$

In Spain, a 10-year prospective cohort study in patients diagnosed with OA of the knee or hip joint revealed a significantly higher average lifetime risk for knee than for hip replacement - 30\% (95\% confidence interval - CI - 25-36\%) versus $14 \%$ (95\% CI 10-19\%). Among the studied risk factors precipitating TKA, early age at the time of diagnosis of knee OA and the increased body mass index (BMI) were identified. ${ }^{17}$ In a German study the OA prevalence among the population aged 60 or older was $21.8 \%$, rising to $31 \%$ in those older than $80 .{ }^{18}$

Due to a significant list of comorbidities in patients undergoing TKA $^{19}$ on one hand and the complex innervation of the knee joint on the other, ${ }^{20}$ postoperative analgesia following the elective knee surgery evolves towards a multimodal approach, combining nerve blocks with the conventional systemic administration of analgesics. ${ }^{21,22}$ Among the several nerve blocks used so far, ${ }^{20,23,24}$ the " 3 -in-1" femoral nerve block (FNB) 25 and the fascia iliaca compartment nerve block (FICNB) ${ }^{26}$ have been most frequently used.

The aim of this clinical trial was to compare these two techniques with the classical systemic analgesic scheme and to compare their postoperative analgesic efficacy between themselves in patients undergoing TKA.

\section{METHODS}

A total of 60 patients of both genders older than 55, scheduled for total knee replacement were included in this randomised clinical study. They were randomised to reach equal gender distributions by means of a block-randomisation method into three groups - MP, NFB and FICNB. The study protocol had been approved by the local Ethics Committee for Trials Involving Human Subjects and followed the principles of the Declaration of Helsinki.

All patient were subjected to TKA under the general balanced anaesthesia and received postoperatively a standard analgesic treatment consisting of morphine 5 or $10 \mathrm{mg}$ q6h iv (a dose of 5 $\mathrm{mg}$ administered to patients weighing up to 60 $\mathrm{kg}$, while the $10 \mathrm{mg}$ dose was reserved for those above that weight limit) and paracetamol $1 \mathrm{~g} \mathrm{q} 6 \mathrm{~h}$ iv. In order to obtain equal gender ratio in every group, patients were block-randomised to one of three groups: (1) standard morphine/paracetamol (MP) group, (2) "3-in-1" femoral nerve block (FNB) group and (3) fascia iliaca compartment nerve block (FICNB) group.

FNB was performed in the inguinal region as a " 3 -in-1" block, as described elsewhere. ${ }^{25,27}$ It included injection of 30-40 ml of a local anaesthetic bupivacaine $0.5 \%$ around the femoral nerve in the inguinal region. For FICNB a technique published earlier ${ }^{26,28}$ was followed. A single injection 30-40 $\mathrm{ml}$ of bupivacaine $0.5 \%$ was administered to anaesthetise the three branches of the lumbar plexus: $n$. cutaneus lateralis, $n$. femoralis and $n$. obturatorius.

The following parameters were registered: basic demographics, pain intensity on the o-10 numeric pain scale (NPS), need for extra doses of analgesics postoperatively, time to the occurrence of such a need, duration of nerve block and cardiovascular parameters.

Statistical analysis was performed by using SPSS version 17.0. Kolmogorov-Smirnov test was used to test the normality of the data dis- 
tribution. For parametric data Student t-test, ANOVA and Tukey post-hoc test were chosen as optimal statistical methods. For non-parametric data Mann-Whitney U test and Kruskal-Wallace test were employed. Probability at $<0.05$ was considered significant.

\section{RESULTS}

The demographic characteristics of all three groups of patients are contained in Table 1.

\begin{tabular}{lllll}
\hline Characteristic & & MP & FNB & FICNB \\
\hline Number of patients & 20 & 20 & 20 \\
\hline ASA status (N) & II & 7 & 6 & 8 \\
& III & 13 & 14 & 12 \\
\hline Gender & Female & 13 & 13 & 13 \\
& Male & 7 & 7 & 7 \\
\hline Age (years) & Mean \pm SD & $72.45 \pm 9.70$ & $72.70 \pm 10.68$ & $70.25 \pm 9.50$ \\
& Range & $57-90$ & $56-88$ & $57-85$ \\
\hline Body weight (kg) & Mean \pm SD & $73.90 \pm 11.58$ & $72.80 \pm 13.0012 .88$ & $75.25 \pm 10.57$ \\
\cline { 2 - 4 } & Range & $56-96$ & $55-101$ & $57-93$ \\
\hline Body height (m) & Mean \pm SD & $1.70 \pm 0.07$ & $1.69 \pm 0.10$ & $1.70 \pm 0.09$ \\
& Range & $1.56-1.84$ & $1.47-1.88$ & $1.51-1.82$ \\
\hline BMl (kg/m $\left.{ }^{2}\right)$ & Mean \pm SD & $25.58 \pm 3.14$ & $25.36 \pm 3.96$ & $26.17 \pm 3.37$ \\
& Range & $20.57-32.91$ & $18.62-35.76$ & $19.27-34.58$ \\
\hline Duration of & Mean \pm SD & $107.45 \pm 15.00$ & $111.25 \pm 15.22$ & $106.25 \pm 17.40$ \\
\hline operation (min) & Range & $87-131$ & $83-135$ & $80-135$ \\
\hline
\end{tabular}

Table 1: Demographic characteristics of patients subjected to total knee arthroplasty.
All three groups of patients were similar regarding the ASA status, age, body weight and height, BMI and the duration of operation.

Mean numeric pain scores for all three groups of patients taken hourly after the end of the operation are contained in Figure 1.

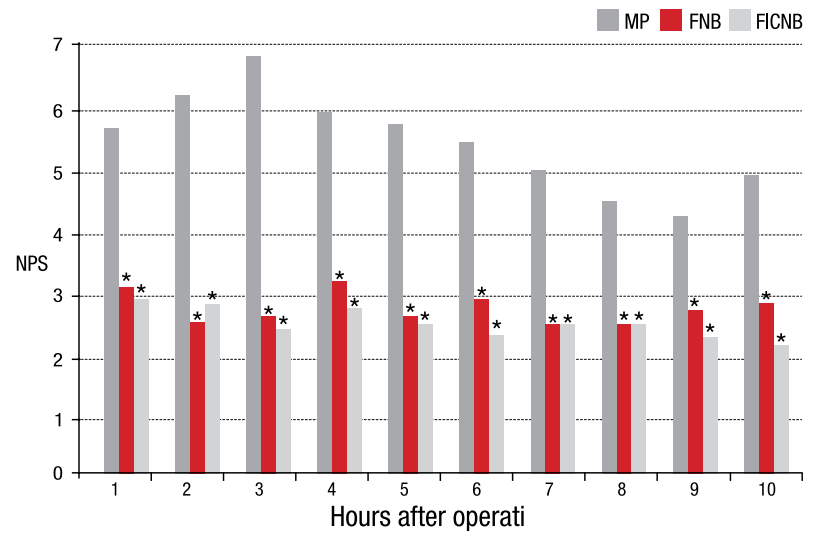

Figure 1: Effect of analgesic regimens on postoperative pain scores in patients after knee alloarthroplasty.

NPS - numeric pain scale, MP - morphine/paracetamol, FNB - femoral nerve block, FICNB - fascia iliaca compartment nerve block; ${ }^{*} p<0.05$ vs the MP group.
It is obvious that the patients in the MP had much higher pain scores than in the other two groups. This difference was the greatest three hours after the end of operation. Thereafter it started to diminish, becoming non-significant only after gth hour. There was no difference in pain scores between the FNB and FICNB.

The time when additional analgesic was needed postoperatively, and the duration of neural blocks are given in Table 2.

In the MP group, the need for additional doses of analgesics occurred much earlier than in the other two groups, while the difference in this time was not significant when the FNB and FICNB groups were compared.

The postoperative use of morphine and paracetamol during the first $24 \mathrm{~h}$ postoperatively in all three groups of patients is shown in Table 3. 
Table 2: Need for additional analgesics and duration of neural block in patients undergoing total knee arthroplasty.

\begin{tabular}{lllll}
\hline & & MP & FNB & FICNB \\
\hline $\begin{array}{l}\text { Time until first } \\
\text { additional dose of } \\
\text { analgesic (h) }\end{array}$ & Mean \pm SD & $4.10 \pm 1.25$ & $7.90 \pm 1.12^{\star}$ & $8.20 \pm 1.58^{*}$ \\
\cline { 2 - 5 } & Range & $2-6$ & $6-10$ & $6-11$ \\
\hline $\begin{array}{l}\text { Duration of nerve } \\
\text { block (h) }\end{array}$ & Mean \pm SD & n/a & $4.60 \pm 1.19$ & $5.10 \pm 0.97$ \\
\cline { 2 - 3 } & Range & n/a & $3-7$ & $4-7$ \\
\hline
\end{tabular}

MP - morphine paracetamol, FNB - femoral nerve block, FICNB - fascia iliaca compartment nerve block ${ }^{*} \mathrm{p}<0.05$ vs the MP group, n/a - not applicable.

Table 3: Additional analgesic consumption in patients undergoing total knee arthroplasty during first $24 \mathrm{~h}$ after the end of operation.

\begin{tabular}{lllll}
\hline & & MP & FNB & FICNB \\
\hline Morphine (mg/ & Mean \pm SD & $5.50 \pm 4.84$ & $1.25 \pm 2.22^{\star}$ & $1.00 \pm 2.05^{*}$ \\
patient/first 24 h) & Range & $0-10$ & $0-5$ & $0-5$ \\
\hline Paracetamol & Mean \pm SD & $4.70 \pm 0.8$ & $2.10 \pm 0.85^{\star}$ & $2.05 \pm 0.94^{*}$ \\
(g/patient/first 24 h) & Range & $4-6$ & $1-4$ & $1-4$
\end{tabular}

MP - morphine paracetamol, FNB - femoral nerve block, FICNB - fascia iliaca compartment nerve block ${ }^{*} \mathrm{p}<0.05$ vs the MP group.

Both in case of morphine and paracetamol, the postoperative consumption was significantly higher in the MP group in comparison with the FNB and FICNB groups. At the same time, the difference between these two groups themselves was not significant.

Changes in systolic blood pressure over the 5 -hour period after the end of operation in all three groups of patients is shown in Figure 2.

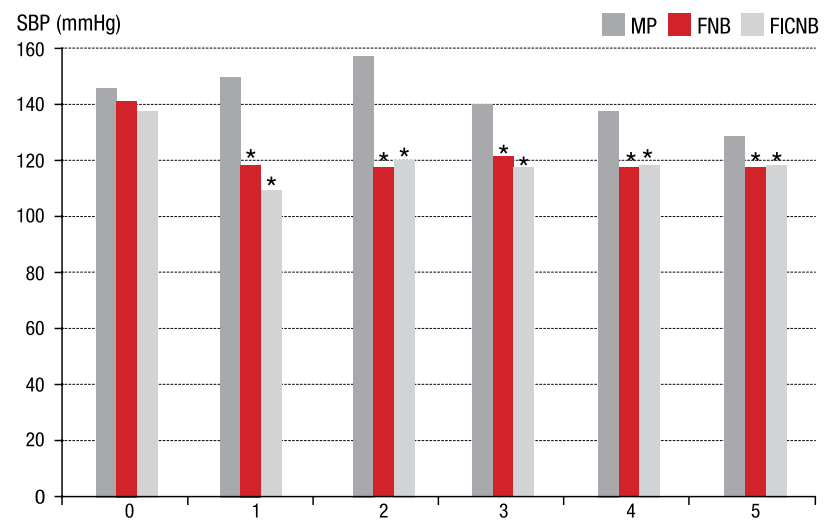

Figure 2: Changes in systolic blood pressure in three groups of patients after total knee arthroplasty.

SBP - systolic blood pressure, MP - morphine/paracetamol, FNB - femoral nerve block, FICNB - fascia iliaca compartment nerve block; ${ }^{*} \mathrm{p}<0.05$ vs the MP group.

It is obvious that at the end of operation values of systolic blood pressure were similar in all three groups. However, in the following hours, they stayed high only in the MP group, while in the FNB and FICNB groups they significantly decreased.

Mean number of episodes of adverse effects is shown in Table 4.

Table 4: Frequency of postoperative adverse effect in patients subjected to total knee arthroplasty (number of episodes /patient).

\begin{tabular}{lllll}
\hline & & MP & FNB & FICNB \\
\hline Nausea & Mean \pm SD & $2.30 \pm 0.98$ & $0.55 \pm 0.69$ & $0.55 \pm 0.69$ \\
& Range & $1-4$ & $0-2$ & $0-2$ \\
\hline Vomiting & Mean \pm SD & $1.25 \pm 0.97$ & $0.10 \pm 0.31^{*}$ & $0.15 \pm 0.37^{*}$ \\
& Range & $0-3$ & $0-1$ & $0-1$ \\
\hline Pruritus & Mean \pm SD & $0.35 \pm 0.49$ & $0.15 \pm 0.37^{*}$ & $0.10 \pm 0.31^{*}$ \\
& Range & $0-1$ & $0-1$ & $0-1$ \\
\hline Sedation & Mean \pm SD & $0.85 \pm 0.37$ & $0.15 \pm 0.37^{*}$ & $0.15 \pm 0.37^{*}$ \\
& Range & $0-1$ & $0-1$ & $0-1$ \\
\hline
\end{tabular}

MP - morphine paracetamol, FNB - femoral nerve block, FICNB - fascia iliaca compartment nerve block ${ }^{*} \mathrm{p}<0.05$ vs the MP group

It is obvious that postoperative nausea, vomiting, pruritus and sedation occur significantly less frequently in FNB and FICNB groups, in comparison with the MP group.

\section{DISSCUSION}

Results of the present study indicate that the two peripheral nerve block procedures, FNB and FICNB, assure significantly less frequent need for rescue analgesic medication, better analgesia, cardiovascular control and adverse effect profile when compared to the morphine/paracetamol regimen. In none of the monitored parameters any difference in efficacy or tolerability between 3-in-1 FNB and FICNB could be found.

Reduction in the pain intensity, judged according to the lower scores on the NPS in comparison with the opioid group, found in the FNB group was in accordance with the results of several other clinical trials. ${ }^{29-36}$ Publications on the postoperative efficacy of FICNB are less abundant, but a meta-analysis unequivocally confirms the results of the present study. ${ }^{37}$ One of the rare available clinical studies publishing the head-tohead comparison between 3-in-1 FNB and FICNB found no difference in the efficacy and safety of these two multi-modal analgesic techniques, ${ }^{34}$ corroborating thus the current results. 
Use of classical opioid-based postoperative techniques is accompanied by classical opioid adverse effects, such as postoperative nausea and vomiting (PONV), pruritus and sedation. In the present study, the decreased need for the rescue morphine administration resulted in diminished frequency and intensity of the morphine adverse effects, which paralleled the findings of the quoted clinical trials. ${ }^{29-31}$ Present results were also similar to the ones obtained in the same clinical settings, but in patients subjected to total hip replacement. ${ }^{38}$

When it comes to pain control during and after TKA, five anaesthetic/analgesic techniques are being used: (1) general anaesthesia (2) spinal anaesthesia, (3) spinal and peripheral anaesthesia, (4) general and spinal anaesthesia and (5) general and peripheral anaesthesia. Although each technique has some advantages and drawbacks, it seems that the combination of intraoperative general balanced anaesthesia and postoperative peripheral anaesthesia (nerve blocks) offers best analgesia and adverse effect profile. ${ }^{23} \mathrm{Neu}-$ ral blocks other than FNB and FICNB are also being used, with similar effects, such as psoas compartment block (PCB) ${ }^{39}$ and adductor canal block (ACB). ${ }^{40}$ These new variations of the multimodal concept of analgesia offer no additional value, in comparison with the FNB and FICNB.

Both morphine and bupivacaine, a long-acting amide local anaesthetic, affect cardiovascular system. Morphine is an opioid obtained from the plant known as opium poppy (Papaver somniferum), while the other currently used opioid analgesics are synthetic. There are many side effects of opioids ${ }^{41}$ and one of the most common effects on the cardiovascular system is prolongation of the QT interval which can lead to torsade de pointes (TDP), kind of the ventricular tachyarrhythmia that can provokes sudden death. ${ }^{22,43}$ The other side effects on the cardiovascular system are bradycardia, histamine release, and rhythm disturbance. Mechanism of action of prolongation of QT interval is associated with prolonged cardiac repolarisation that is initiated by rapid outflow of potassium $(\mathrm{K}+)$, through the cardiac rapid-rectifying $\mathrm{K}+$ channel. ${ }^{42}$

Racemic bupivacaine (Marcaine) is potentially cardiotoxic, having depressant electrophilic effects on the heart, especially in patients with compromised cardiac function. It depresses the intracardiac conduction velocity and cardiac contractility. By blocking $\mathrm{Na}+$ and $\mathrm{K}+$ channels it causes the prolongation of the PR and QT intervals in the electrocardiogram. Levobupivacaine is less cardiotoxic than the racemic bupivacaine ${ }^{44}$ Cardiotoxicity is usually not a problem in low single doses of bupivacaine, as the ones used in this clinical trial.

\section{CONCLUSION}

Multimodal anaesthesia, combining intraoperative general balanced anaesthesia and postoperative local anaesthetic-induced peripheral nerve blocks, such as FNB and FICNB, enables better analgesia and fewer adverse effects than the morphine/paracetamol postoperative regimen.

\section{CONFLICT OF INTEREST}

None.

\section{ACKNOWLEDGEMENTS}

None.

\section{REFERENCES}

1. Sharma L, Kapoor D, Issa S. Epidemiology of osteoarthritis: an update. Curr Opin Rheumatol 2006;18:14756.

2. Hvidberg MF, Johnsen SP, Davidsen M, Ehlers L. A Nationwide study of prevalence rates and characteristics of 199 chronic conditions in Denmark. PharmacoEconomics - Open https://doi.org/10.1007/s41669019-0167-7.

3. Lee S, Kwon Y, Lee N, Bae KJ, Kim J, Park S, et al. The Prevalence of Osteoarthritis and Risk Factors in the Korean Population: The Sixth Korea National Health and Nutrition Examination Survey (VI-1, 2013). Korean J Fam Med 2019;40(3):171-175.

4. Bryan S, Goldsmith LJ, Davis JC, Hejazi S, MacDonald V, McAllister P, et al. Revisiting patient satisfaction following total knee arthroplasty: a longitudinal observational study. BMC Muskuloskelet Disord 2018 Nov 30;19(1):423. doi: 10.1186/s12891-018-2340-z.

5. Kontio T, Viikari-Juntura E, Solovieva S. To what extent do education and physical work load factors explain occupational differences in disability retirement 
due to knee OA? A nationwide register-based study in Finland. BMJ Open 2018; 8:e023057. doi:10.1136/bmjopen-2018-023057.

6. Mahomed NN, Barrett J, Katz JN, Baron JA, Wright $\mathrm{J}$, Losina E. Epidemiology of total knee replacement in the United States Medicare population. J Bone Joint Surg Am 2005;87(6):1222-8.

7. Kopec JA, Rahman MM, Berthelot JM, Le Petit C, Aghajanian J, Sayre EC, et al. Descriptive epidemiology of osteoarthritis in British Columbia. Canada J Rheumatol 2007;34(2):386-93.

8. Kopec JA, Rahman MM, Sayre EC, Cibere J, Flanagan WM, Aghajanian J, et al. Trends in physician-diagnosed osteoarthritis incidence an an administrative database in British Columbia, Canada, 1996-1997 through 20032004. Arthrit Rheum-Arthr 2008;59(7):929-34.

9. Wallace IJ, Worthington S, Felson DT, Jurmain RD, Wren KT, Maijanen H, Woods RJ, Lieberman DE. Knee osteoarthritis has doubled in prevalence since the mid-20th century. Proc Natl Acad Sci U S A 2017;114(35):9332-6.

10. Mirmaroofi N, Ghahramanian A, Behshid M, Jabbarzadeh F, Onyeka TC, Asghari-Jafarabadi M, et al. Relationship between self-efficacy and pain control in Iranian women with advanced knee osteoarthritis. Niger J Clin Pract 2019;22(4):460-8.

11. Clynes MA, Jameson KA, Edwards MH, Cooper C, Dennison EM. Impact of osteoarthritis on activities of daily living: does joint site matter? Aging Clin Exp Res 2019;31:1049-56.

12. Lespasio MJ, Piuzzi NS, Husni ME, Muschler GF, Guarino AJ, et al. Knee osteoarthritis: a primer. Perm J 2017; 21:16-183.

13. Tennant A, Fear J, Pickering A, Hillman M, Cutts A, Chamberlain MA. Prevalence of knee problems in the population aged 55 years and over: identifying the need for knee arthroplasty. Br Med J 1995;310(6990):12913 .

14. Canadian Institute for Health Information (CIHI). Hip and Knee Replacements in Canada, 2016-2017: Canadian joint replacement registry annual report. Ottawa: CIHI; 2018 [Available from: https://www. cihi.ca/en/ canadian-joint-replacement-registry-cjrr].

15. Kurtz S, Ong K, Lau E, Mowat F, Halpern M. Projections of primary and revision hip and knee arthroplasty in the United States from 2005 to 2030. J Bone Joint Surg Am 2007; 89:780-5.

16. Maempel JF, Riddoch F, Calleja N, Brenkel IJ. Longer hospital stay, more complications, and increased mortality but substantially improved function after knee replacement in older patients. Acta Orthop 2015;86(4):451-6.

17. Burn E, Murray DW, Hawker GA, Pinedo-Villanueva $\mathrm{R}$, Prieto-Alhambra D. Lifetime risk of knee and hip replacement following a GP diagnosis of osteoarthritis: a real-world cohort study. Osteoarthritis and Cartilage, https://doi.org/10.1016/j.joca.2019.06.004.

18. Postler A, Ramos AL, Goronzy J, Günther KP, Lange T,
Schmitt J, et al. Prevalence and treatment of hip and knee osteoarthritis in people aged 60 years or older in Germany: an analysis based on health insurance claims data. Clin Interv Aging 2018;13:2339-49.

19. Jämsen E, Peltola M, Eskelinen A, Lehto MUK. Comorbid diseases as predictors of survival of primary total hip and knee replacements: a nationwide register-based study of 96754 operations on patients with primary osteoarthritis. Ann Rheum Dis 2013; 72:19751982.

20. Kukreja P, Feinstein J, Kalagara HK, Huntley SR, Lee SR, Naranje S, et al. A summary of the anatomy and current regional anesthesia practices for postoperative pain management in total knee arthroplasty. Cureus 2018 Jun 7; 10(6):e2755. doi: 10.7759/cureus.2755.

21. Chaubey D, et al. Comparison of continuous femoral nerve block versus local infiltration analgesia as a postoperative analgesia in unilateral total knee arthroplasty. J Clin Diagnostic Res 2017; 11(7):UC13-UC16.

22. Soffin EM, Memtsoudis SG. Anesthesia and analgesia for total knee arthroplasty. Minerva Anestesiol 2018; 84(12):1406-12.

23. Greimel F, Maderbacher G, Baier C, Keshmiri A, Schwarz T, Zeman F, et al. Multicenter cohort-study of 15326 cases analyzing patient satisfaction and perioperative pain management: general, regional and combination anesthesia in knee arthroplasty. Sci Rep 2018 Feb 27; 8(1):3723. doi: 10.1038/s41598-018-22146-7.

24. Li Y, Li A, Zhang Y. The efficacy of combined adductor canal block with local infiltration analgesia for pain control after total knee arthroplasty: A meta-analysis. Medicine (Baltimore). 2018 Dec; 97(49):e13326. doi: 10.1097/MD.ooooooooooo13326.

25. Winnie AP, Ramamurthy S, Durrani Z. The inguinal paravascular technic of lumbar plexus anesthesia: the "3-in-1 block". Anesth Analg 1973; 52(6):989-96.

26. Dalens B, Vanneuville G, Tanguy A. Comparison of the fascia iliaca compartment block with the 3 -in-1 block in children. Anesth Analg. 1989 Dec;69(6):705-13.

27. Rosenblatt RM. Continuous femoral anesthesia for lower extremity surgery. Anesth Analg 1980;59(8):631-2.

28. Chudinov A, Berkenstadt H, Salai M, Cahana A, Perel A. Continuous psoas compartment block for anesthesia and perioperative analgesia in patients with hip fractures. Reg Anesth Pain Med 1999;24(6):563-8.

29. 29. Özen M, Inan N, Tümer F, Uyar A, Baltac B. The effect of 3-in-1 femoral nerve block with ropivacaine $0.375 \%$ on postoperative morphine consumption in elderly patients after total knee replacement surgery. Agri 2006;18(4):44-50.

30. Seet E, Leong WL, Yeo AS, Fook-Chong S. Effectiveness of 3 -in-1 continuous femoral block of differing concentrations compared to patient controlled intravenous morphine for post total knee arthroplasty analgesia and knee rehabilitation. Anaesth Intensive Care 2006;34(1):25-30.

31. Park CK, Cho CK, Lee JH, Shin HH. Optimizing the dose of intrathecal morphine when combined with 
continuous 3-in-1 nerve block after total knee replacement. Korean J Anesthesiol 2009 Jul;57(1):69-77.

32. 32. Paul JE, Arya A, Hurlburt L, Cheng J, Thabane L, Tidy A, et al. Femoral nerve block improves analgesia outcomes after total knee arthroplasty: a meta-analysis of randomized controlled trials. Anesthesiology 2010;113(5):1144-62.

33. Tulgar S, Selvi O, Senturk O, Serifsoy TE, Sanel S, Meydaneri S. Evaluation of analgesic regimens in total knee arthroplasty, retrospective study. North Clin Istanb 2017;4(2):124-30.

34. Wang X, Sun Y, Wang L, Hao X. Femoral nerve block versus fascia iliaca block for pain control in total knee and hip arthroplasty. A meta-analysis from randomized controlled trials. Medicine (Baltimore) $2017 \mathrm{Jul}$; 96(27):e7382. doi: 10.1097/MD.oooooooooo007382.

35. Novello-Siegenthaler A, Hamdani M, Iselin-Chaves I, Fournier R. Ultrasound-guided continuous femoral nerve block: a randomized trial on the influence of femoral nerve catheter orifice configuration (six-hole versus end-hole) on post-operative analgesia after total knee arthroplasty. BMC Anesthesiology 2018 Dec 19;18(1):191. doi: 10.1186/s12871-018-0648-8.

36. Zhang J, Yuan Y, Zhang Y, Wang Y. Clinical effects of single femoral nerve block in combination with general anesthesia on geriatric patients receiving total knee arthroplasty. Pak J Med Sci 2018;34(1):43-8.

37. Zhang P, Li J, Song Y, Wang X. The efficiency and safety of fascia iliaca block for pain control after total joint arthroplasty. A meta-analysis. Medicine (Baltimore) 2017 Apr;96(15):e6592 doi: 10.1097/ MDoooooooooooo6592.
38. Lončar-Stojiljković D, Stojiljković MP, Golijanin R, Novaković Bursać S, Škrbić R. Comparative postoperative analgesia with femoral nerve block " 3 -in-1" and with fascia iliaca compartment nerve block after hip alloarthroplasty. Med Čas (Krag) 2016;50(1):12-6.

39. Touray ST, de Leeuw MA, Zuurmond WW, Perez RS. Psoas compartment block for lower extremity surgery: a meta-analysis. Br J Anaesth 2008;101(6):750-60.

40. Tan Z, Kang P, Pei F, Shen B, Zhou Z, Yang J. A comparison of adductor canal block and femoral nerve block after total-knee arthroplasty regarding analgesic effect, effectiveness of early rehabilitation, and lateral knee pain relief in the early stage. Medicine (Baltimore) 2018 Nov;97(48):e13391. doi: 10.1097/ MDoooooooooooo13391.

41. Bowdle TA. Adverse effects of opioid agonists and agonist-antagonists in anaesthesia. Drug Saf 1998; 19(3):173-89.

42. Barkin RL, Barkin SJ, Barkin DS. Propoxyphene (dextropropoxyphene): a critical review of a weak opioid analgesic that should remain in antiquity. Am J Ther 2006;13:534-42.

43. Behzadi M, Joukar S, Beik A. Opioids and cardiac arrhythmia: a literature review. Med Princ Pract 2018;27(5):401-14.

44. Sathyanarayana LA, Heggeri VM, Simha PP, Narasimaiah S, Narasimaiah M, Subbarao BK. Comparison of epidural bupivacaine, levobupivacaine and dexmedetomidine in patients undergoing vascular surgery. J Clin Diagn Res 2016 Jan;10(1):UC13-7. doi: 10.786o/ JCDR/2016/17344.7079. 